\title{
What Happens to Hydrophobic Interactions during Transfer from the Solution to the Gas Phase? The Case of Electrospray-Based Soft Ionization Methods
}

\author{
Konstantin Barylyuk, ${ }^{1}$ Roman M. Balabin, ${ }^{1}$ Dan Grünstein, ${ }^{2,3}$ Raghavendra Kikkeri, ${ }^{2,3}$ \\ Vladimir Frankevich, ${ }^{1}$ Peter H. Seeberger, ${ }^{2,3}$ Renato Zenobi ${ }^{1}$ \\ ${ }^{1}$ Department of Chemistry and Applied Biosciences, ETH Zurich, Wolfgang-Pauli Strasse 10, 8093 Zurich, Switzerland \\ ${ }^{2}$ Department of Biomolecular Systems, Max Planck Institute of Colloids and Interfaces, Am Mühlenberg 1, 14476 Potsdam, \\ Germany \\ ${ }^{3}$ Institute of Chemistry and Biochemistry, Free University Berlin, Arnimallee 22, 14195 Berlin, Germany
}

\begin{abstract}
The disappearance of the hydrophobic effect in the gas phase due to the absence of an aqueous surrounding raises a long-standing question: can noncovalent complexes that are exclusively bound by hydrophobic interactions in solution be preserved in the gas phase? Some reports of successful detection by mass spectrometry of complexes largely stabilized by hydrophobic effect are questionable by the presence of electrostatic forces that hold them together in the gas phase. Here, we report on the MS-based analysis of model supramolecular complexes with a purely hydrophobic association in solution, $\beta$-cyclodextrin, and synthetic adamantyl-containing ligands with several binding sites. The stability of these complexes in the gas phase is investigated by quantum chemical methods (DFT-M06). Compared with the free interaction partners, the inclusion complex between $\beta$-cyclodextrin and adamantyl-containing ligand is shown to be stabilized in the gas phase by $\Delta G=9.6 \mathrm{kcal} \mathrm{mol}^{-1}$. The host-guest association is mainly enthalpy-driven due to strong dispersion interactions caused by a large nonpolar interface and a high steric complementarity of the binding partners. Interference from other types of noncovalent binding forces is virtually absent. The complexes are successfully detected via electrospray ionization mass spectrometry, although a high dissociation yield is also observed. We attribute this pronounced dissociation of the complexes to the collisional activation of ions in the atmospheric interface of mass spectrometer. The comparison of several electrospray-based ionization methods reveals that cold spray ionization provides the softest ion generation conditions for these complexes.
\end{abstract}

Key words: Electrospray ionization mass spectrometry, Non-covalent complexes, Hydrophobic interactions, Van der Waals-London dispersion interactions, Gas phase

Electronic supplementary material The online version of this article (doi:10.1007/s13361-011-0118-8) contains supplementary material, which is available to authorized users.

Correspondence to: Renato Zenobi; e-mail: zenobi@org.chem.ethz.ch

\section{Introduction}

Soft ionization methods in mass spectrometry (MS), such $S_{\text {as }}$ the electrospray ionization (ESI), give access to a broad variety of analytes with complex covalent structures, including biomolecules and biopolymers $[1,2]$. In recent years, the focus has shifted from simple analysis of complex 
organic and biological molecules to probing of higher-order structures that are stabilized by noncovalent interactions. ESI-MS, with certain modifications, proved to be an efficient tool for the analysis of supramolecular assemblies and biomolecular noncovalent complexes. The most common approach for the analysis of biomolecular noncovalent complexes utilizes electrospraying analytes under nearnative conditions (aqueous buffer, controlled $\mathrm{pH}$, room temperature) via a nanoflow ESI interface (nano-ESI [3]) [4-6].

Increasing interest in using MS to study structures, where noncovalent bonding is important, inspired the invention of novel variants of electrospray technique that promised to be even softer. Electrosonic spray ionization (ESSI), a variant of ESI that employs a supersonic nebulizing gas flow, has recently been introduced by Cooks and co-workers as a very gentle method for generating ions of folded proteins and protein noncovalent complexes [7]. Jecklin et al. reported a comparative study on the softness of different electrospraybased methods (ESSI, nano-ESI, and ESI) showing that ESSI is capable of better preserving protein-ligand noncovalent complexes [8]. Cold-spray ionization (CSI), a variant of electrospray that operates at low temperatures, has been applied for the analysis of labile organic compounds, such as transition metal coordination complexes, Grignard reagents, and supramolecules, e.g., amino acid and nucleotide clusters and host-guest complexes [9].

Since these methods are all claimed to probe solutionphase properties, the question arises to what extent the structure and supramolecular organization of analytes in the gas-phase environment of a mass spectrometer reflects that in solution. It is therefore important to preserve all the interactions that stabilize the structure of the analyte upon ionization and transfer to the gas phase. While certain supramolecular forces (e.g., Coulomb attraction/repulsion, ion-dipole, and dipolar interactions) become even stronger in vacuum, hydrophobic interactions are difficult to preserve during ionization and ion transfer $[10,11]$. Indeed, the binding energy of a methane contact pair in water is estimated to be only $1.5 \mathrm{kcal} \mathrm{mol}^{-1}[12,13]$. On the other hand, multiple hydrophobic interactions can become very strong and greatly contribute to the overall system stability. For instance, up to $70 \%$ of the total stabilization energy of protein tertiary structure originates from the collapse of protein hydrophobic core [14-16]. However, hydrophobic interactions are driven by the gain in entropy of released solvent molecules during complexation [17, 18]. In MS, analytes are desolvated during delivery into the gas phase, i.e., there is no more gain in entropy. Strictly speaking, hydrophobic interactions degenerate into van der WaalsLondon dispersion interactions in the gas phase.

A number of studies report on more or less successful analyses of noncovalent complexes where hydrophobic forces are considered to play a dominant role for the stability [19-33]. Liu et al. [19, 20] showed the preservation of hydrophobically stabilized complex between bovine $\beta$ - lactoglobulin and its natural ligands, fatty acids, for the fraction of ESI-generated ions. However, hydrogen bonding between the protein chain and fatty acid carboxylic group also contributed significantly to complex stabilization [19]. Further investigation of this system revealed a greater kinetic stability of gaseous complexes between the fatty acids and $\beta$ lactoglobulin due to a significantly higher activation barrier, as compared with the hydrated complexes [20]. Although the authors attribute this difference in the kinetic stability of the complexes to solvation effects, the increase in the activation energy for complex dissociation might also originate from electrostatic interactions or a steric hindrance between the protein and ligands in the gas phase.

Gas-phase dissociation of proton-bound dimers of fatty acids, as well as of alkali metal cation-bound dimers and trimers of fatty acid methyl esters was found to be affected by hydrophobic interactions between their aliphatic chains $[21,22]$. The complexes containing double bonds in aliphatic chains dissociated more easily than those with fully saturated fatty acid tails. The authors explained this finding by the disturbance of hydrophobic interactions between aliphatic chains caused by the conformational restrictions imposed by double bonds. However, they did not account for the effect of such conformational restriction on the collision cross section of the complexes in the dissociation experiments.

Another model system to study the preservation of hydrophobic interactions in the gas phase is cyclodextrin. Cyclodextrins (CDs) are cyclic oligosaccharides consisting of $\alpha$-1,4-linked glucose residues. In this ring-shaped structure, all hydroxyl groups point outwards, so that the interior of the ring is hydrophobic while the exterior is hydrophilic, rendering CDs water-soluble. CDs allow for the formation of inclusion complexes with various nonpolar molecules in polar solvents [34]. Several studies have reported on the detection of inclusion complexes between CDs and aromatic or aliphatic guests, amino acids, peptides, and drugs by ESIMS [23-33]. However, in these studies, guest molecules bore at least one polar group that pointed out of the cavity and enabled hydrogen bonding or dipole-dipole interactions with hydroxyls of the $\mathrm{CD}$ rim. These forces become stronger in the gas phase due to the electrostatic nature and most probably dominate the complex stabilization in the absence of solvent. A large fraction of nonspecific adducts in the mass spectra of CDs bound to aliphatic ligands is a consequence of such electrostatic attraction between binding partners in the gas phase [23-25].

In the examples described above, the dominant stabilizing forces thus switched from hydrophobic interactions in solution to electrostatic attraction or hydrogen bonding in the gas phase. An unambiguous conclusion on the stability of hydrophobically bound noncovalent complexes in the gas phase therefore cannot be drawn, since species found in the gas phase are stabilized by non-specific interactions substituting the real driving force of complexation in the solution phase. 
To adequately address the question whether hydrophobically stabilized complexes can be preserved in the gas phase, a better model system in which dispersion interactions are the major complex-stabilizing factor in the gas phase is required. Recently, Seeberger et al. described a series of synthetic supramolecular complexes that are stabilized solely by hydrophobic interactions in solution (Scheme 1) [35]. The complexes RuCD2, RuCD4, and RuCD6 consist of synthetic guest ligands with a controlled number of $\mathrm{CD}$ binding sites (two, four or six; Scheme 1: 2-4, respectively) and $\beta$-cyclodextrin (Scheme 1: 1) host molecules [35]. In aqueous solution, the adamantyl residues, which are attached to the ruthenium-containing core of a ligand through amide linkers, intercalate into the inner cavity of CDs solely due to the hydrophobic effect. The formation of inclusion complex was confirmed by $1 \mathrm{D}-{ }^{1} \mathrm{H}-\mathrm{NMR}$ and $2 \mathrm{D}$-nuclear Overhauser enhancement (NOE)-NMR spectroscopy [35]. Although the hydrophobic effect governs the complexation in solution, inspection of this system suggests a significant enthalpy gain even in the absence of solvent due to the large area of interacting nonpolar surfaces and the steric complementarity between the adamantyl and CD cavity (see Table 1 and the 3 section) [34].

The goal of the present study was to directly probe the stability of hydrophobically bound noncovalent complexes under ESI-MS conditions. We report on the MS analysis of RuCD2, RuCD4, and RuCD6 model complexes using several electrospray-based soft ionization methods. Conventional ESI, nano-ESI, ESSI, and CSI are compared in terms of their softness of ionization for these model complexes. A complementary theoretical study of a host-guest complex mimicking the binding site in the RuCDs is performed in order to estimate their stability in the gas phase and investigate the nature of binding force. Our results suggest that in the RuCDs hydrophobic interactions degenerate into dispersion interactions in the gas phase, which, however, suffice to stabilize the complex.

While several groups reported successful detection of hydrophobic complexes under ESI-MS conditions, the nature of the stabilizing forces that hold binding partners

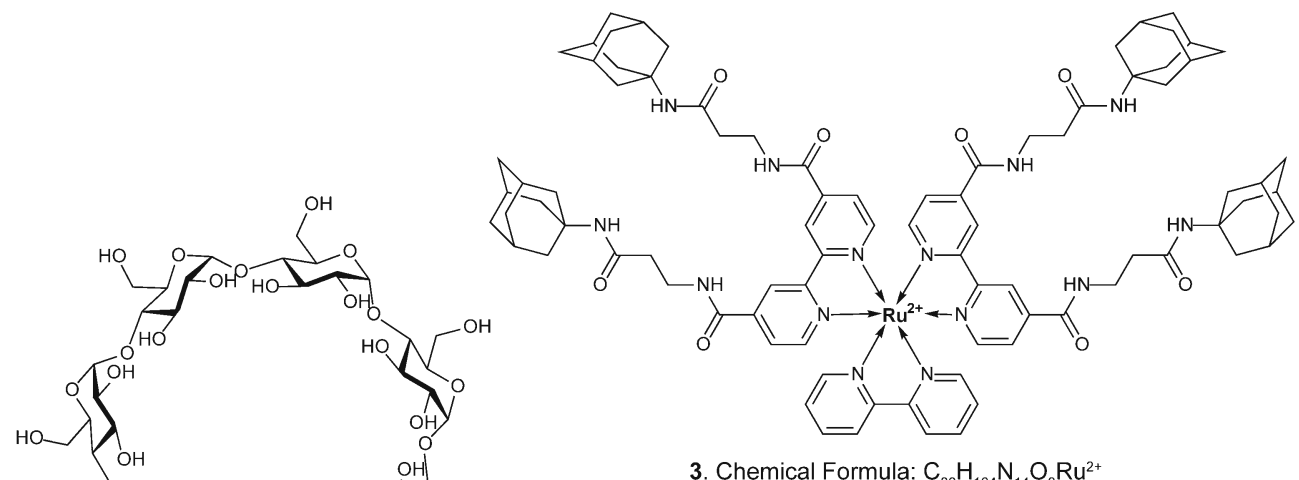

Exact mass: 1562.72
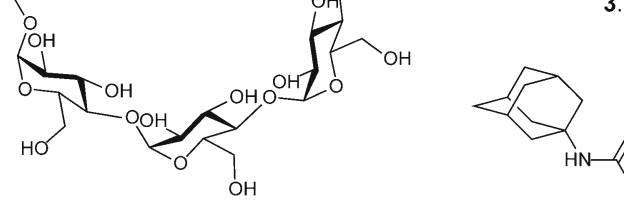

1. Chemical Formula: $\mathrm{C}_{42} \mathrm{H}_{70} \mathrm{O}_{35}$ Exact mass: 1134.37

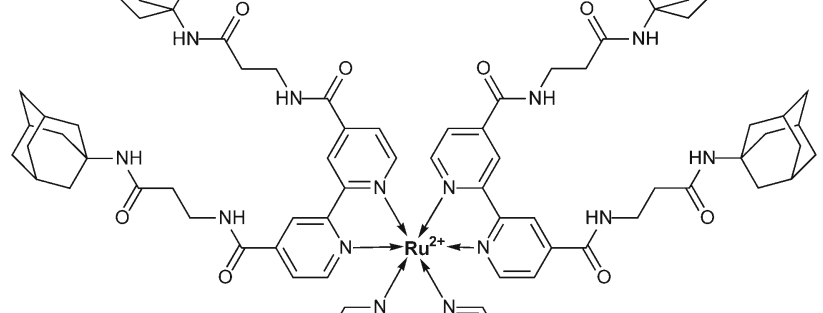

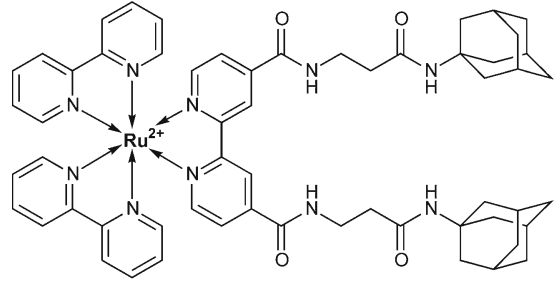

2. Chemical Formula: $\mathrm{C}_{58} \mathrm{H}_{64} \mathrm{~N}_{10} \mathrm{O}_{4} \mathrm{Ru}^{2+}$

Exact mass: 1066.41

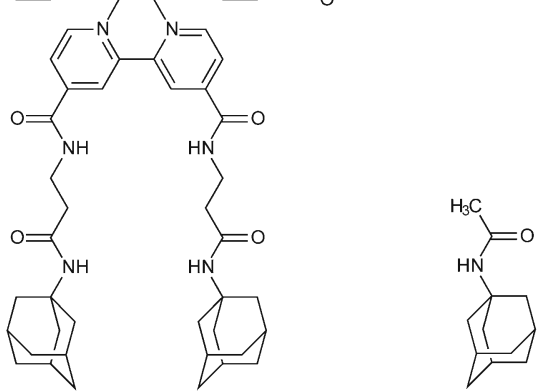

4. Chemical Formula: $\mathrm{C}_{114} \mathrm{H}_{144} \mathrm{~N}_{18} \mathrm{O}_{12} \mathrm{Ru}^{2+} \quad$ 5. AdNAc Exact mass: 2059.02

Scheme 1. Chemical structures of the investigated compounds. RuCD2 $=\mathbf{2}+2 \times \mathbf{1}, \mathrm{MW}=3336.23$. RuCD4 $=\mathbf{3}+4 \times \mathbf{1}, \mathrm{MW}=$ 6102.84. $\mathrm{RuCD} 6=\mathbf{4}+6 \times \mathbf{1}, \mathrm{MW}=8869.45$ 
together has never been investigated in detail. Therefore, we carried out a combined experimental and theoretical study of model supramolecules in order to answer the following questions: can hydrophobically bound complexes be preserved in the gas phase without rearrangement of the stabilizing interactions and what are the prerequisites for that?

\section{Methods}

\section{Mass Spectrometry Measurements}

Supramolecular complexes (RuCD2, RuCD4, and RuCD6; see Scheme 1) were synthesized as described in [35], and dissolved in ultra-pure water to give a final concentration of $10-100 \mu \mathrm{M}$.

Mass spectra were recorded on a hybrid quadrupole-time of flight instrument (Q-TOF Ultima, Micromass, Manchester, UK) equipped with a Z-spray interface (Waters, Manchester, UK). Several ion sources were employed to generate ions, including a conventional ESI source (Waters, Manchester, UK), an automated chip-based nano-ESI robot (Nanomate model 100; Advion Biosciences, Ithaca, NY, USA), as well as home-built ESSI and CSI sources.

In ESI experiments, a voltage of $3.5 \mathrm{kV}$ was applied to the ESI probe, and samples were delivered with a flow rate of $2 \mu \mathrm{L} \min ^{-1}$ via a syringe pump (Harvard Apparatus, Holliston, MA, USA); no sheath or desolvation gas was used, and the declustering temperature was varied in the range of $21-150{ }^{\circ} \mathrm{C}$. The nano-ESI source was operated at a voltage of $1.3-1.5 \mathrm{kV}$, and 5 bar backing pressure was used to assist sample flow, which was set to $200 \mathrm{~nL} \mathrm{~min}{ }^{-1}$; the source temperature was kept at $21^{\circ} \mathrm{C}$.

The design of our home-built ESSI source can be found elsewhere [7]. Briefly, it consisted of a T-piece (Swagelok; BGB Analytik AG, Böckten, Switzerland), an outer and inner fused silica capillary (inner diameters of 375 and $50 \mu \mathrm{m}$, respectively; BGB Analytik AG, Switzerland). A high voltage of $3.5 \mathrm{kV}$ was applied to the ESSI emitter and samples were delivered at a flow rate of $5 \mu \mathrm{L} \mathrm{min}{ }^{-1}$ by a syringe pump; a nitrogen pressure of 10-20 bar was used. The ESSI probe was mounted on a XYZ-stage with a distance to the mass spectrometer inlet of 100-200 mm.

The CSI source was built analogously to the one described in [9]. Samples were dissolved in 50\% aqueous methanol (HPLC grade; Fluka Chemie AG, Buchs, Switzerland) to a final concentration of $10-100 \mu \mathrm{M}$ and delivered at $5 \mu \mathrm{L} \min ^{-1}$ by a syringe pump to a modified ESI probe operated at 3.5-4.5 kV. Dry nitrogen was used as a sheath and desolvation gas to cool down the probe and the ESI plume area; it was delivered at flow rate of $200-500 \mathrm{~L} \mathrm{~min}^{-1}$ via coiled metal tubes (length approximately $3.5 \mathrm{~m}, 7-10$ turns with the diameter approximately $0.1 \mathrm{~m}$ ), which passed through the reservoir with liquid nitrogen. The source temperature was controlled by a thermocouple and adjusted in the range from -40 to $+20{ }^{\circ} \mathrm{C}$ by changing the number of coil turns submerged into liquid nitrogen and/or heating the source.

The sampling cone and the first ion guide (RF lens 1) voltages in all experiments were optimized in the range of 100-150 V and 150-200 V, respectively, in order to achieve the most efficient transmission of the desired ions. The quadrupole transmission mode was tuned to cover the $\mathrm{m} / \mathrm{z}$ range of 300-8000. Collision-induced dissociation (CID) inside a hexapole collision cell was used in MS/MS experiments. Argon (analyzer penning pressure readout $4.5 \times 10^{-5}$ mbar) was used as a collision gas. The collision energy was varied in the range of $10-50 \mathrm{eV}$. CsI clusters generated from the infusion of a $2 \mathrm{~g} \mathrm{~L}^{-1}$ solution of CsI (puriss; Fluka Chemie AG, Buchs, Switzerland) in 50\% aqueous isopropanol (HPLC grade $\geq 99.9 \%$ ) were used for mass calibration.

\section{Data Processing}

Mass spectra were acquired using the MassLynx software (ver. 4.0, Waters, Manchester, UK). A single mass spectrum accumulation time of $1 \mathrm{~s}$ was used, and a total of 100 spectra were typically accumulated to obtain a representative mass spectrum with a sufficient signal-to-noise ratio. Data processing and evaluation was performed using the MATLAB software (2010a; The MathWorks, Natick, MA, USA). Areas under peaks corresponding to ions of assembled, partially and fully disassembled RuCD complexes were used to calculate the survival yield of every species.

\section{Quantum Chemistry Calculations}

The calculations reported here were done within the framework of density functional theory (DFT) using the M06 and M06L, as well as B3LYP and B97 density functionals, and a version of the latter with explicit dispersion correction (B97D) [36, 37]. The M06/M06L functional is specially parameterized to include dispersion interactions - an important bonding factor in the complexes studied here [36]. A Pople-type 6-31G(d) basis sets [38] was used throughout the study. The Gaussian 09 suite of programs [39] was used for most calculations reported. The calculations were carried out according to procedures described in [40]. The geometries of all molecules and complexes reported were fully optimized at the M06L/6-31G(d) level of theory. The energy difference is reported relative to the sum of the individual binding partners. The RRHO approximation was used for evaluating thermodynamic properties. Different conformations of amide bond were considered, but they were found to be of no importance for the relative stability of the supramolecular complexes.

The resulting structures of the inclusion complexes were visualized using the UCSF Chimera software package [41]. The interface areas (Table 1) were calculated based on surfaces created with the surfnet tool [42]. Images were 
Table 1. Energetic Parameters of Model Supramolecular Host-Guest Complexes between $\beta$-cyclodextrin and N-adamantylacetamide

\begin{tabular}{|c|c|c|c|c|c|c|c|}
\hline \multirow[t]{2}{*}{ Complex arrangement (see Figures 3 and 4) } & \multicolumn{3}{|c|}{ Inclusion complexes } & \multicolumn{4}{|c|}{ Nonspecific adducts } \\
\hline & $3 a$ & $3 b$ & $3 \mathrm{c}$ & $4 a$ & $4 \mathrm{~b}$ & $4 \mathrm{c}$ & $4 d$ \\
\hline$\Delta G_{300 \mathrm{~K}}, \mathrm{kcal} \mathrm{mol}^{-1}$ & -3.8 & -9.4 & -2.9 & -1.1 & 4.6 & 11.3 & 20.3 \\
\hline$\Delta H_{300 \mathrm{~K}}, \mathrm{kcal} \mathrm{mol}^{-1}$ & -23.5 & -29.1 & -23.0 & -18.9 & -15.0 & -8.4 & 0.2 \\
\hline$-T \Delta S_{300 \mathrm{~K}}, \mathrm{kcal} \mathrm{mol}^{-1}$ & 19.7 & 19.7 & 20.2 & 17.8 & 19.6 & 19.7 & 20.1 \\
\hline$\Delta E, \mathrm{kcal} \mathrm{mol}^{-1}$ & -28.2 & -33.7 & -20.0 & -25.4 & -19.7 & -13.0 & -9.9 \\
\hline Interface area, $\AA^{2}$ & 221.7 & 201.0 & 215.8 & 150.8 & 133.3 & 153.1 & 139 \\
\hline
\end{tabular}

produced with the POV-ray software (ver. 3.6; Persistence of Vision Pty. Ltd., Williamstown, Victoria, Australia).

\section{Results and Discussion}

\section{Stoichiometry of Complexes}

In ESI-MS experiments, RuCD complexes produced doubly charged ions with very characteristic isotope pattern due to the presence of $\mathrm{Ru}^{2+}$, allowing for a clear assignment of the peaks. Figure 1a shows the nano-ESI mass spectrum of RuCD2. Besides the peak at $m / z 1667.4$ corresponding to the assembled complex (Figure 1a, inset), signals of the partially assembled complex and the bare ligand $\mathbf{2}$ (Scheme 1) were also registered (peaks at $\mathrm{m} / \mathrm{z} 1100.4$ and 533.2, respectively). RuCD4 and
RuCD6 complexes showed similar behavior (Figure 1b and c, respectively). The signal intensity of the RuCD4 ion $(\mathrm{m} / \mathrm{z}$ 3050.1) was quite low and the RuCD6 complex ( $\mathrm{m} / \mathrm{z}$ 4432.6) was registered with a signal-to-noise ratio of approximately 6 (insets in Figure $1 \mathrm{~b}$ and $\mathrm{c}$ ). Numerous additional peaks appearing in the spectra (Figure 1a, b and c) were due to the presence of impurities in the samples. For instance, cationized cyclodextrins and oligosaccharides produced strong signals in the measured $m / z$ range. No non-specific RuCD associates with higher CD-to-ligand stoichiometric ratio were found in mass spectra in the higher $m / z$ range.

The stoichiometry of the RuCD complexes was further probed by MS/MS measurements (Figure 1d, e and f). At low collision energies, the major dissociation channel was the loss of single neutral CD. With increasing collision energy,
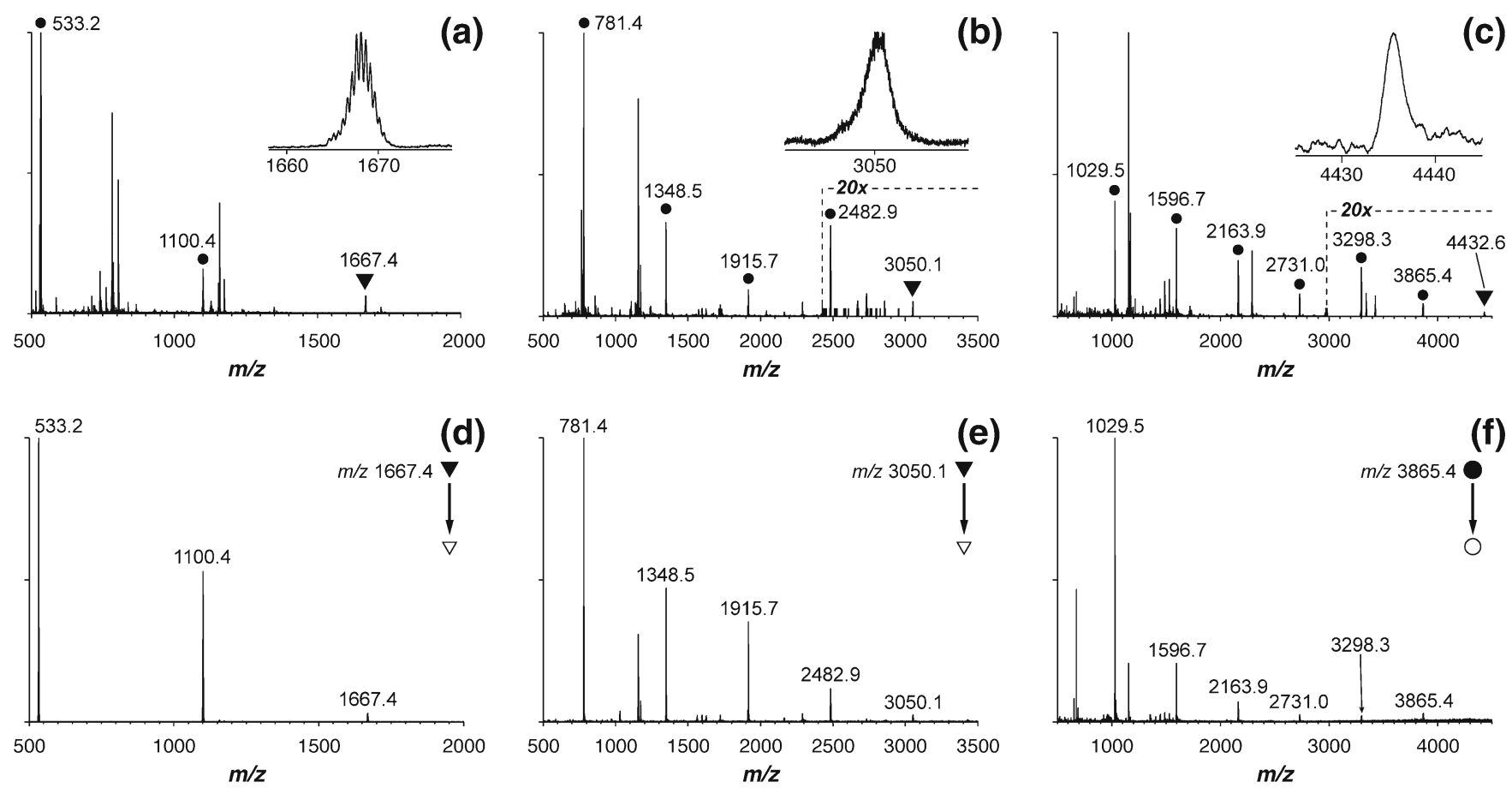

Figure 1. Nano-ESI mass spectra (a)-(c) and CID-MS/MS spectra (d-f) of RuCD complexes. Peaks corresponding to the fully assembled RuCD2 (a), RuCD4 (b), and RuCD6 (c) complexes are marked with triangles and are additionally shown as zoomed views [(a)-(c), insets]. Signals from partially and fully disassembled complexes are marked with circles. Unmarked peaks are due to sample impurities, e.g., cationized cyclodextrin ions and their fragments. The intensity scale was magnified 20 -fold in the mass range marked with the dashed line in the mass spectra of RuCD4 and RuCD6 [(b) and (c), respectively]. lons of assembled RuCD2 and RuCD4 complex were mass-selected in the quadrupole stage and subjected to CID inside the collision cell [(d) and (e), respectively]. In the case of RuCD6, the ion of incompletely assembled RuCD6 complex (with five cyclodextrins bound) was selected and dissociated (f) 
detachment of multiple CD molecules was observed. However, these channels were very close in energy, since the ions of partially assembled complexes readily fragmented via the loss of neutral $\mathrm{CD}$ in $\mathrm{MS}^{3}$ experiments, at similar collision energy offset. In the case of RuCD6, the signal intensity of the fully assembled complex was not enough to record a MS/MS spectrum, and the ion at $\mathrm{m} / \mathrm{z} 3865.4$ corresponding to the complex of $5 \mathrm{CD}$ molecules bound to the ligand $\mathbf{4}$ was massselected and fragmented instead (Figure 1f).

To prove the specificity of detected RuCD complexes the following control experiment was performed. A 10-fold molar excess of CD was added to the solution of RuCD2 and the resulting mixture was analyzed by nano-ESI-MS. In the case of a non-specific association between $\mathrm{CD}$ and ligand $\mathbf{2}$ the increase of $C D$ concentration would result in formation of complexes with three or more CDs attached. However, this was not the case, as can clearly be seen from Figure 2. While a number of weak, nonspecific complexes of cationized CDs became visible in the mass spectrum, no adducts of $\mathrm{RuCD} 2$ with one or two more $\mathrm{CD}$ molecules was found (Figure 2b, m/z 2235.6 and 2803.1, respectively). Very weak peaks with a signal-to-noise ratio close to three were found at $m / z 1490.4,1498.1$, and 1503.4, which might be attributed to the presence of triply charged ions of $\mathrm{RuCD} 2$ forming adducts with $\mathrm{CD}^{+},[\mathrm{CD}+\mathrm{Na}]^{+}$, and $[\mathrm{CD}+$
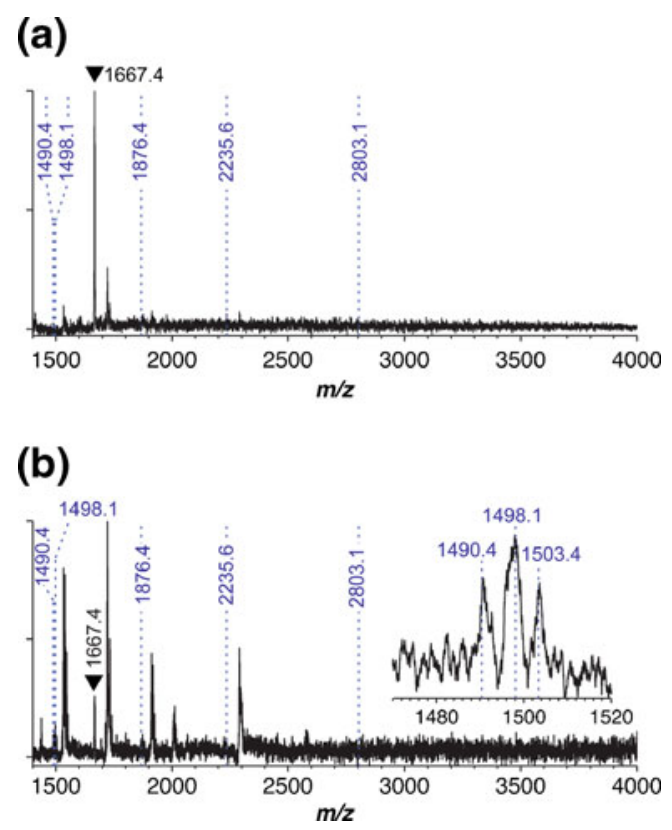

Figure 2. Nano-ESI mass spectra of $10 \mu \mathrm{M}$ aqueous solution of RuCD2 (a) and of the same solution containing 10-fold molar excess of CD (b). A peak corresponding to the fully assembled RuCD2 complex is marked with the black triangle. An expected position of peaks corresponding to non-specific adducts between ligand 2 (see Scheme 1) and CDs is indicated with blue dashed lines and corresponding $\mathrm{m} / \mathrm{z}$ values. The inset shows the magnified region of the mass spectrum around $\mathrm{m} / \mathrm{z} 1500$ (b), where negligible peaks of nonspecific adducts of RuCD2 and cationized CDs were found (see Discussion section in the text)
$\mathrm{K}]^{+}$, respectively. However, the signal intensity was too low to perform MS/MS experiments, which, together with the lack of isotopic resolution, prevented us from unambiguously assigning these peaks. Given that the concentration of analytes in this control experiment was quite high $(\sim 100 \mu \mathrm{M})$, the presence of these small peaks in the mass spectrum is more consistent with the non-specific association of RuCD2 and cationized CDs in the ESI plume rather than with non-specific complexation between the CDs and ligand $\mathbf{2}$ in solution [31, 43].

Similar experiments where a 10 -fold molar excess of CD was added to RuCD4 and RuCD6 were also carried out. Again, many peaks of nonspecific adducts between CDs and metal cations were found in the spectra. However, no peaks corresponding to non-specific adducts of RuCD4 and RuCD6 with CDs were registered.

\section{Stability of the Complexes in Solution and in the Gas Phase}

Three reasons can lead to the low signal intensities of the assembled complexes in the mass spectra: low complex stability in solution, low complex stability in the gas phase, and poor complex survival during passage through the ESI interface. The NMR results allow us to rule out the dissociation of complexes in solution [35]. There was no unbound CD found in ${ }^{1} \mathrm{H}-\mathrm{NMR}$ spectra, and NOESY spectra showed a clear evidence for the close proximity of the adamantyl protons to those of $\mathrm{CD}$. In the gas phase, we expect the complexes to be stable due to a decent gain in enthalpy from dispersion interactions. Indeed, the large nonpolar interface and steric complementarity allow for the formation of multiple contacts between the adamantyl residue and the inner cavity of $\mathrm{CD}$. Although individual interactions might be weak, their sum provides strong stabilization overall. Moreover, dispersion interactions are enhanced in vacuum due to the stronger electrostatic term.

We ran quantum chemistry calculations on the model supramolecular assembly of CD (1 on Scheme 1) and Nadamantylacetamide (AdNAc; 5 on Scheme 1) in order to (1) prove the stability of model complexes in the gas phase; (2) support the statement that the observed signals originate from the inclusion complexes described in solution and are not due to artificial adducts formed during the ionization process; (3) show that the major force that stabilizes the complexes in the gas phase is a strong dispersion attraction and not a hydrogen bond or any kind of electrostatic interaction, as for instance in cases reported by Franski et al. [21, 22]. The position of the adamantyl guest was optimized, and several relative orientations of binding partners were tested (Figure 3): a tight inclusion complexes with the guest entering from the 6'-OH and the 2',3'-OH rims of $\mathrm{CD}$, respectively (Figure $3 \mathrm{a}$ and $\mathrm{b}$ ), and an inclusion complex with an intermolecular hydrogen bond (Figure 3c). It is noteworthy that the optimized geometries of the model inclusion complexes closely resembled the available crystal 

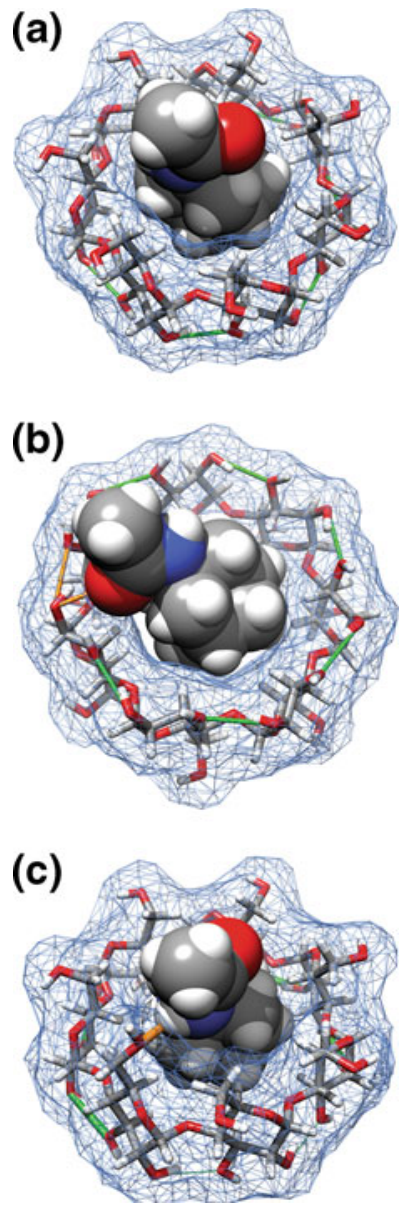

Figure 3. Relative orientations of $\mathrm{N}$-adamantylacetamide (AdNAc; 5 on Scheme 1) and $\beta$-cyclodextrin (CD; 1 on Scheme 1) within the model supramolecular assembly probed by quantum chemistry calculations. AdNAc atoms are shown as spheres with van der Waals radii, CD is represented by stick model with the van der Waals surface superimposed (blue mesh). The entrance of AdNAc guest into the $\mathrm{CD}$ cavity from the 6'-OH rim (a), (c) and from the $2^{\prime}, 3^{\prime}-\mathrm{OH}$ rim (b) was explored. Hydrogen bonds within the constraints of a $0.4 \mathrm{~nm}$ inter-atom distance and a $20^{\circ}$ bond angle were monitored (shown by green lines), as well as those only partially satisfying these restrictions [orange lines, (b) and (c)]

structures of CD complexes with adamantyl ligands [44-46]. The possibility of intermolecular hydrogen bond formation without inclusion of the adamantyl moiety into the cavity of CD was also explored (Figure 4). The choice of starting geometries for the nonspecific assemblies was dictated by the requirement of forming of at least one hydrogen bond. However, our intention was to test the relative orientations that maximize the number of hydrogen bonds since the binding partners contain numerous hydrogen bond donors and acceptors. Remarkably, only the structure shown in Figure $4 \mathrm{~b}$ allowed for two hydrogen bonds between the 6'$\mathrm{OH}$ groups of two adjacent glucose residues and the amide group of AdNAc. In the other three cases, the formation of only one hydrogen bond was successful, most probably due to steric hindrance (Figure $4 \mathrm{a}, \mathrm{c}$ and d).

The M06 and M06L methods were employed, which are specifically designed to account for systems with dispersion interactions and show fairly accurate results of the binding energy calculation in these systems, with an error of $\sim 2 \mathrm{kcal} \mathrm{mol}^{-1}$ [47]. The 6-31 G(d) basis set was chosen as a compromise between accuracy and computational costs since no significant gain in accuracy was expected for a larger basis set [48]. The binding energy $(\Delta E)$ of tight inclusion complexes (Figure $3 \mathrm{a}$ and $\mathrm{b}$ ) was estimated to be about $-30 \mathrm{kcal} \mathrm{mol}^{-1}$ (Table 1). In comparison, the structure with an intermolecular hydrogen bond between the amide proton of AdNAc and the oxygen atom of the 6'-OH group from $\mathrm{CD}$ (Figure 3c) was $8 \mathrm{kcal} \mathrm{mol}^{-1}$ less stable. Among the nonspecific complexes, the strongest binding was found for the assembly of AdNAc positioned parallel to the plane of the $\mathrm{CD}$ ring from the 2', $3^{\prime}-\mathrm{OH}$ side (Figure $4 \mathrm{a}$ ), giving $\Delta E=-25.4 \mathrm{kcal} \mathrm{mol}^{-1}$ (Table 1). Quite a remarkable binding energy $\left(-19.7 \mathrm{kcal} \mathrm{mol}^{-1}\right.$, Table 1) was also found for the structure with two hydrogen bonds (Figure 4b). The other two non-specific adducts (Figure 4c and d) gave much smaller binding energies of only about $-10 \mathrm{kcal} \mathrm{mol}^{-1}$ (Table 1 ).

All the relative orientations of binding partners that we tested resulted in the formation of energetically favorable complexes $(\Delta E<0)$. Calculation of the enthalpy and Gibbs free energy allowed us to draw a conclusion on the driving force of complexation and the relative stability of these complexes. From the Table 1 it can clearly be seen that the complexes have a strong negative entropy term $(\Delta S<0)$. To compensate the loss of entropy, a large gain in enthalpy of association $\left(>20 \mathrm{kcal} \mathrm{mol}^{-1}\right)$ is needed, which in this case can only be achieved via multiple van der Waals contacts between the bulky adamantyl moiety and the inner cavity of CD. Since the enthalpy of $-\mathrm{OH}{ }^{\cdots} \mathrm{O}=$ and $-\mathrm{NH}^{\cdots} \mathrm{O}-$ hydrogen bonds typically amounts to less than $5 \mathrm{kcal} \mathrm{mol}^{-1}$ [49], at least 4 hydrogen bonds are required to compensate the loss of entropy found for the model assemblies. However, AdNAc and CD do not allow for the formation of 4 hydrogen bonds. In agreement with our estimations, the fairly large positive Gibbs free energy allows us to rule out the formation of three nonspecific adducts (Figure 4b, c and d) in the gas phase. The assembly in which AdNAc enters the CD cavity from the 2',3'-OH rim (Figure $3 b$ ) appeared to be the most stable among the remaining four complexes (Figures 3a, b and c and 4a).

We selected the most stable model complex between AdNAc and CD (Figure 3b) and ran binding energy calculations using the B97D (with correction for dispersion interactions) and B97 (without dispersion correction) density functionals in order to estimate the contribution of dispersion interactions to the overall stabilization energy. When the dispersion interactions were accounted for, the binding energy of the complex amounted to $-36.1 \mathrm{kcal} \mathrm{mol}^{-1}$, in reasonable agreement to the result of M06 calculation (-33.7 $\mathrm{kcal} \mathrm{mol}^{-1}$, also corrected for dispersion interactions; see Table 1). In contrast, switching off the dispersion term 

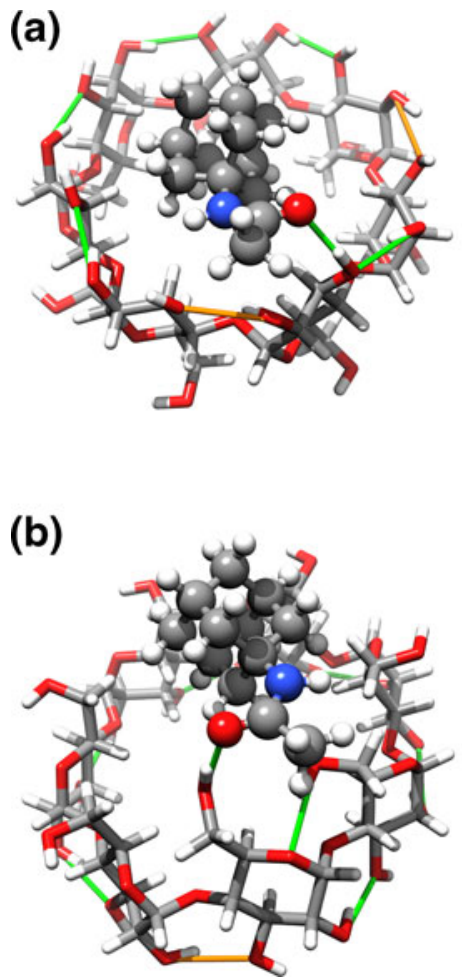
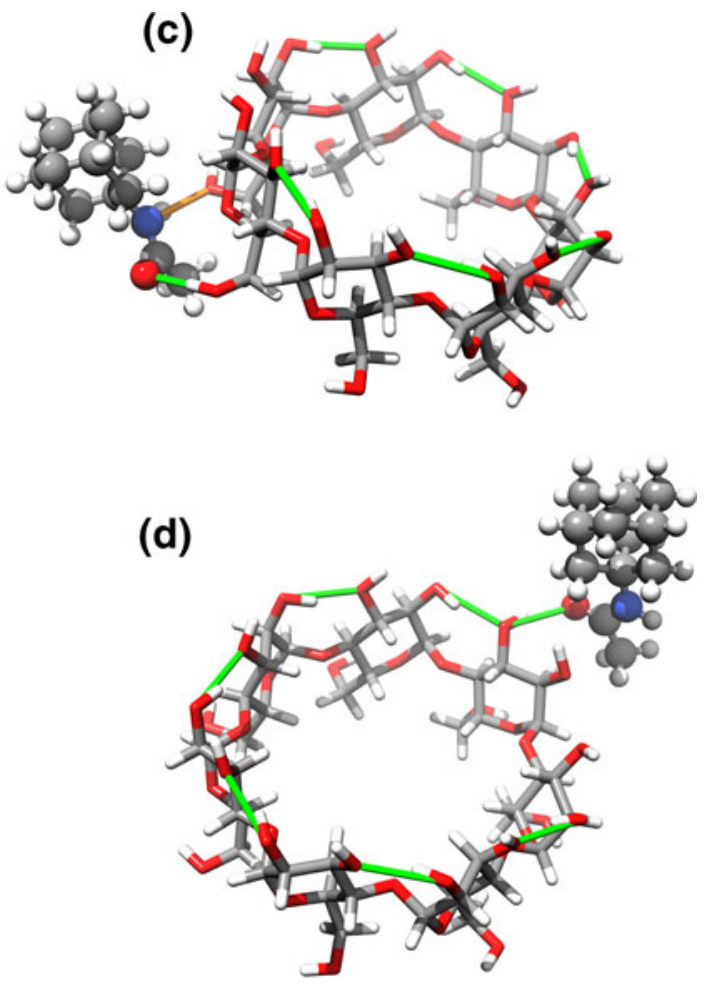

Figure 4. Geometries of model nonspecific supramolecular assemblies between N-adamantylacetamide (AdNAc; 5 on Scheme 1) and $\beta$-cyclodextrin (CD; 1 on Scheme 1) studied by quantum chemical calculations. AdNAc is shown as a ball-andstick model, CD is represented in sticks. Hydrogen bonds are marked as in Figure 3

(pure B97) resulted in only $-6.0 \mathrm{kcal} \mathrm{mol}^{-1}$ of binding energy. Therefore, $\sim 30 \mathrm{kcal} \mathrm{mol}^{-1}$ or $83 \%$ of the total stabilization energy of this complex (Figure $3 \mathrm{~b}$ ) comes from multiple dispersion interactions, and the rest can be attributed to some spurious electrostatic interactions (e.g., see the orange "pseudobonds" shown in Figure $3 b$ and c). The latter should, however, be significantly weaker due to steric factors: CD fits tightly around the bulky adamantyl moiety, resulting in steric constraints that prevent hydroxyl and amide dipoles from assuming the correct orientation to form a strong hydrogen bond or dipole-dipole contact (Figure S1). The same steric factor hinders the host-guest association, so if the complex dissociated in the gas phase, its re-association would require a lot of collisions/time, since only a very small fraction of host-guest relative orientations would lead to a reactive collision.

The results of quantum chemistry calculations reported here clearly indicate that the RuCD complexes should be stable in the gas phase due to strong van der Waals attraction between the CD inner cavity and the adamantyl moiety of the ligands. It must therefore be the ESI interface that is responsible for the dissociation of complexes.

\section{Stability of the Complexes as a Function of Ion Source Configuration}

ESI is operated at atmospheric pressure while the mass spectrometer requires high vacuum to analyze and detect ions. Electrospray-generated ions thus have to pass several differential pumping chambers to reach the analyzer and detector. Due to a steep pressure drop at the interface of atmosphere and the first low-pressure chamber, ions undergo supersonic expansion and acquire high kinetic energy. However, there are still a lot of neutral gas molecules around and the accelerated ions collide with them, which results in gradual activation of ions. This "in-source collision-induced activation" can be very strong and will lead under certain circumstances to pronounced dissociation. Even covalent bonds ( 40-60 kcal mol $\left.{ }^{-1}[50,51]\right)$ can be disrupted, not to appeal of relatively weak supramolecular forces, such as dispersion interactions. Low signal intensities of assembled RuCD complexes along with strong signals of fragment ions might therefore be attributed to the processes during ionization and transfer into the mass spectrometer.

There are several ways to soften the ion transfer. Generally speaking, voltage and pressure gradients should be lowered in order not to accelerate ions too much and induce collisional cooling rather than collision-induced activation of ions [51]. However, in the Q-TOF instrument, the so-called Z-spray interface is used in the ion source that requires for higher ion acceleration in order to facilitate transmission. The heavier the ions, the higher the voltage gradient that is needed to transfer ions through the Z-spray interface. In the case of the quite heavy RuCD ions, we had to use a fairly high voltage difference between the sampling cone and the first ion guide. The pressure drop can be reduced by "spoiling" the vacuum 
(a)

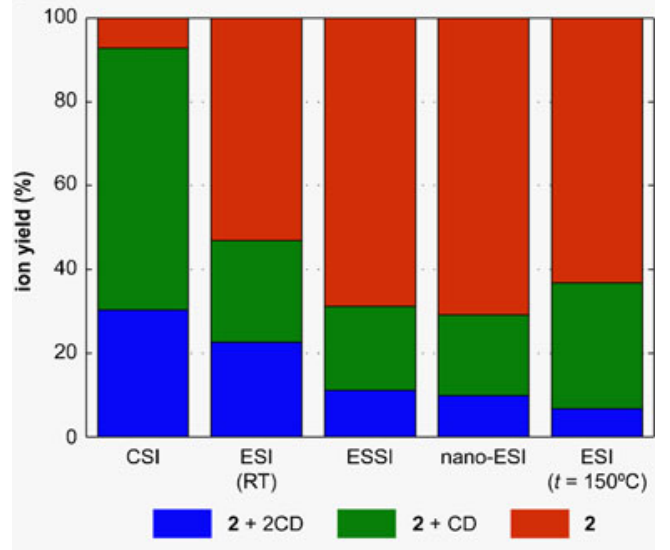

(b)

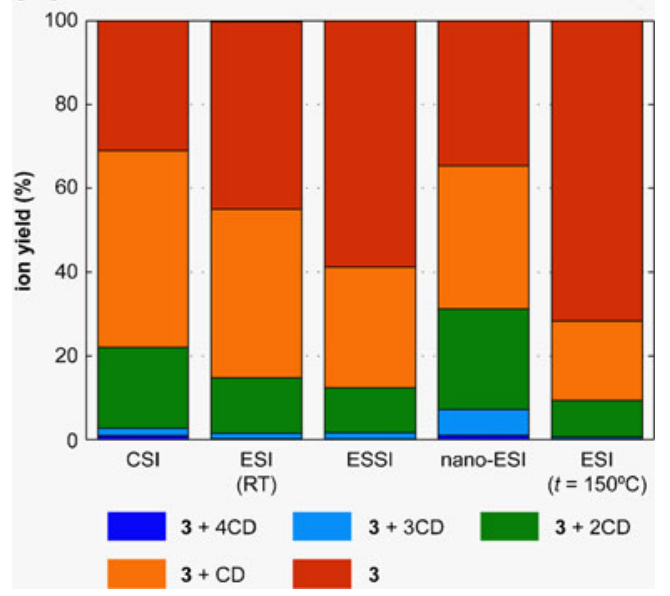

Figure 5. Influence of the ion source configuration on the survival of RuCD2 (a) and RuCD4 (b) complexes during ionization and transfer through the atmospheric interface of mass spectrometer. The fractions of fully assembled, partially assembled, and dissociated complexes in mass spectra are plotted in stacks of bar plots and color-coded, as described below each plot. The stoichiometry of assemblies is described using the nomenclature from Scheme 1. CD $=\beta$ cyclodextrin; RT = room temperature

inside the first pumping stage. This trick is often used in the analysis of protein noncovalent complexes [5]. In our experiments, there was only minor or zero gain in signal of the assembled RuCD complexes when the pressure inside the first pumping region was elevated, so we worked at normal pressure values in this region.

The ion generation itself, of course, affects the survival of intact complexes in such MS experiments. Jecklin et al. have demonstrated that protein-ligand noncovalent complexes are better preserved in ESSI compared with conventional ESI and nano-ESI [8], although in their later work authors showed almost equal ion internal energy distribution for these electrospray-based methods [52]. In the case of noncovalent assemblies that rely on dispersion interactions, such as RuCD complexes, we expected an even more pronounced dependence of complex survival on the ion generation process.

To investigate the softness of electrospray processes for hydrophobically stabilized supramolecular complexes, we compared the ion yields in MS experiments where ions were generated by several ESI-based methods (Figure 5, Figure S2). In conventional ESI experiments, ion yields of the assembled RuCD2 were relatively low and fragments dominated the spectra (Figure 5a, Figure S2a). Upon increasing the temperature in the source the dissociation of complex became more pronounced, revealing a clear dependence of the complex stability on the operating parameters of the electrospray. Thermal dissociation is known to occur for supramolecular complexes, and we expected them to be better preserved upon ionization and transfer to the gas phase at low temperature. Indeed, in CSI experiments, the fraction of assembled RuCD2 was significantly higher compared with conventional ESI. Although the dissociation of RuCD2 down to the bare ligand $\mathbf{2}$ was low, the yield of partially assembled complex was still high. We also compared the results of ESI at different temperatures with those obtained with other soft ionization methods, namely nano-ESI and ESSI [3, 6, 7]. Surprisingly, RuCD2 showed high dissociation rates in these experiments (Figure 5a, Figure S2a). For larger complexes, e. g. RuCD4, the result was very similar (Figure 5b, Figure S2b). With increasing source temperature the rate of dissociation became higher; conversely, RuCD4, as well as partially assembled complexes, were better preserved at low temperature in CSI-MS. In ESSI, RuCD4 dissociation was quite pronounced, while nano-ESI showed better result with high degree of preservation of the intermediate assemblies.

In electrospray, ions are produced as a consequence of Coulomb explosions of charged droplets [43]. Recently, Chingin et al. demonstrated that gas-phase ions are produced by electrospray in abundance already at ambient pressure [53] and validated the ion evaporation hypothesis for small ions [54, 55]. Several steps of solvent evaporation and subsequent droplets fission are, however, required prior to ion evaporation. The initial size of droplets emitted from the electrospray tip in conventional ESI, nano-ESI, and ESSI is very different: $10-100 \mu \mathrm{m}, 0.15 \mu \mathrm{m}$, and $3.5 \mu \mathrm{m}$, respectively [7, 43, and references therein]. In nano-ESI, the initial droplet size $(0.15 \mu \mathrm{m})$ is already quite small, so that ion evaporation from the droplet surface can become operative from the very beginning of the ion drift path. In ESSI, the supersonic sheath gas flow provides very efficient nebulization of electrosprayed solvent. Again, ions are produced at early stages after droplets leave the emitter tip. As soon as the gas-phase ions are generated, they pass the voltage gradient and undergo numerous collisions with neutral gas molecules. These collisions result in partial conversion of kinetic energy to ion internal energy. In other words, ions are gradually heated [51]. In case of thermally unstable supramolecular complexes, this slow activation is sufficient to induce their dissociation. The higher the source temper- 
ature, the more internal energy the ions gain, resulting in a high degree of in-source fragmentation. Conversely, at lower temperatures, collisions are less energetic and the internal energy uptake is reduced.

In-source ion activation and dissociation thus explains the high fragment yield in experiments reported herein. Since RuCD complexes were quite bulky, they obviously have large collisional cross-section, which renders collisional activation and internal energy uptake very efficient. Lower survival yields of larger complexes, such as RuCD6, were most probably the consequence of larger collisional crosssection. On the other hand, relatively high yields of intermediate assemblies might be attributed to the redistribution of excess energy over a higher number of degrees of freedom available in larger complexes.

\section{Conclusions}

In the present work, the model noncovalent complexes RuCD2, RuCD4, and RuCD6 based solely on hydrophobic interactions in solution were studied by soft ionization MS. Our experimental data demonstrate that such complexes can be preserved in the gas phase under ESI conditions, provided that the van der Waals attraction between binding partners is strong enough to compensate the loss of entropy. Quantum chemical calculations were employed to confirm the stability of model complexes in the gas phase and to investigate the nature of interactions in the host-guest assembly. In the gas phase, the stabilizing forces in the binding site reduce to mainly dispersion interactions $\left(\Delta E=-30.1 \mathrm{kcal} \mathrm{mol}^{-1}\right)$, with only minor contribution from other types of interactions $\left(\Delta E=-6.0 \mathrm{kcal} \mathrm{mol}^{-1}\right)$. The high steric complementarity of the binding partners together with the large contact area result in a high binding energy $\left(\Delta G=-9.4 \mathrm{kcal} \mathrm{mol}^{-1}\right.$ per binding site). The large size and the solubility of the complexes in water allow them to mimic the behavior of large protein ions during the ionization and ion transmission processes. All these features render the RuCD complexes a unique model system to study the preservation of hydrophobically stabilized noncovalent assemblies by MS.

Fully assembled RuCD2, RuCD4, and RuCD6 were successfully detected by ESI-MS, along with fragment ions of lower CD-to-ligand stoichiometric ratio. We attribute this fact to in-source dissociation of the complexes rather than to their lack of stability in the solution or in the gas phase. A comparison of several electrospray-based ionization techniques revealed a clear dependence of complex survival on the parameters of the ion source. The source temperature had the highest impact on the stability of complexes under ESI conditions. Cold spray ionization provided the softest conditions for ionization of these model complexes.

\section{Acknowledgments}

The authors gratefully acknowledge financial support from the Swiss National Foundation (SNF grant 200020-124663), from the Max-Planck Society, and from the European Union
FP7 (CARMUSYS). Quantum chemistry calculations were carried out on the Obelix cluster at the Competence Center for Computational Chemistry, ETH Zurich.

\section{References}

1. Yamashita, M., Fenn, J.B.: Electrospray ion-source-another variation on the free-jet theme. J. Phys. Chem. 88(20), 4451-4459 (1984)

2. Fenn, J.B., Mann, M., Meng, C.K., Wong, S.F., Whitehouse, C.M.: Electrospray ionization for mass-spectrometry of large biomolecules. Science 246(4926), 64-71 (1989)

3. Wilm, M., Shevchenko, A., Houthaeve, T., Breit, S., Schweigerer, L., Fotsis, T., Mann, M.: Femtomole sequencing of proteins from polyacrylamide gels by nano-electrospray mass spectrometry. Nature 379(6564), 466-469 (1996)

4. Heck, A.J.R., van den Heuvel, R.H.H.: Investigation of intact protein complexes by mass spectrometry. Mass Spectrom. Rev. 23(5), 368-389 (2004)

5. Hernandez, H., Robinson, C.V.: Determining the stoichiometry and interactions of macromolecular assemblies from mass spectrometry. Nat. Protoc. 2(3), 715-726 (2007)

6. van den Heuvel, R.H., Heck, A.J.R.: Native protein mass spectrometry: from intact oligomers to functional machineries. Curr. Opin. Chem. Biol. 8(5), 519-526 (2004)

7. Takats, Z., Wiseman, J.M., Gologan, B., Cooks, R.G.: Electrosonic spray ionization. A gentle technique for generating folded proteins and protein complexes in the gas phase and for studying ion-molecule reactions at atmospheric pressure. Anal. Chem. 76(14), 4050-4058 (2004)

8. Jecklin, M.C., Touboul, D., Bovet, C., Wortmann, A., Zenobi, R.: Which electrospray-based ionization method best reflects protein-ligand interactions found in solution? A comparison of ESI, nanoESI, and ESSI for the determination of dissociation constants with mass spectrometry. J. Am. Soc. Mass Spectrom. 19(3), 332-343 (2008)

9. Yamaguchi, K.: Cold-spray ionization mass spectrometry: principle and applications. J. Mass Spectrom. 38(5), 473-490 (2003)

10. Robinson, C.V., Chung, E.W., Kragelund, B.B., Knudsen, J., Aplin, R. T., Poulsen, F.M., Dobson, C.M.: Probing the nature of noncovalent interactions by mass spectrometry. A study of protein-CoA ligand binding and assembly. J. Am. Chem. Soc. 118(36), 8646-8653 (1996)

11. Wu, Q., Gao, J., Joseph-McCarthy, D., Sigal, G.B., Bruce, J.E., Whitesides, G.M., Smith, R.D.: Carbonic anhydrase-inhibitor binding: from solution to the gas phase. J. Am. Chem. Soc. 119(5), 1157-1158 (1997)

12. Rick, S.W., Berne, B.J.: Free energy of the hydrophobic interaction from molecular dynamics simulations: the effects of solute and solvent polarizability. J. Phys. Chem. B 101(49), 10488-10493 (1997)

13. Li, J.L., Car, R., Tang, C., Wingreen, N.S.: Hydrophobic interaction and hydrogen-bond network for a methane pair in liquid water. Proc. Natl Acad. Sci. U.S.A. 104(8), 2626-2630 (2007)

14. Nicholls, A., Sharp, K.A., Honig, B.: Protein folding and associationinsights from the interfacial and thermodynamic properties of hydrocarbons. Proteins Struct. Funct. Genet. 11(4), 281-296 (1991)

15. Vondrasek, J., Bendova, L., Klusak, V., Hobza, P.: Unexpectedly strong energy stabilization inside the hydrophobic core of small protein rubredoxin mediated by aromatic residues: correlated $\mathrm{Ab}$ initio quantum chemical calculations. J. Am. Chem. Soc. 127(8), 2615-2619 (2005)

16. Agashe, V.R., Shastry, M.C.R., Udgaonkar, J.B.: Initial hydrophobic collapse in the folding of barstar. Nature 377(6551), 754-757 (1995)

17. Pratt, L.R., Chandler, D.: Theory of hydrophobic effect. J. Chem. Phys. 67(8), 3683-3704 (1977)

18. Pratt, L.R.: Molecular theory of hydrophobic effects: "she is too mean to have her name repeated.". Annu. Rev. Phys. Chem. 53, 409-436 (2002)

19. Liu, L., Bagal, D., Kitova, E.N., Schnier, P.D., Klassen, J.S.: Hydrophobic protein-ligand interactions preserved in the gas phase. J. Am. Chem. Soc. 131(44), 15980 (2009)

20. Liu, L., Michelsen, K., Kitova, E.N., Schnier, P.D., Klassen, J.S.: Evidence that water can reduce the kinetic stability of protein-hydrophobic ligand interactions. J. Am. Chem. Soc. 132(50), 17658-17660 (2010)

21. Franski, R., Gierczyk, B., Kozik, T.: Tandem mass spectrometry experiments support the existence of hydrophobic interactions in the gas phase. Rapid Commun. Mass Spectrom. 22, 2747-2749 (2008)

22. Franski, R., Gierczyk, B., Schroeder, G., Franska, M., Wyrwas, B.: Do hydrophobic interactions exist in the gas phase? Rapid Commun. Mass Spectrom. 22(8), 1339-1343 (2008) 
23. Gabelica, V., Galic, N., De Pauw, E.: On the specificity of cyclodextrin complexes detected by electrospray mass spectrometry. J. Am. Soc. Mass Spectrom. 13(8), 946-953 (2002)

24. Cunniff, J.B., Vouros, P.: False positives and the detection of cyclodextrin inclusion complexes by electrospray mass spectrometry. J. Am. Soc. Mass Spectrom. 6(5), 437-447 (1995)

25. Kralj, B., Smidovnik, A., Kobe, J.: Mass spectrometric investigations of $\alpha$ - and $\beta$-cyclodextrin complexes with ortho-, meta-, and para-coumaric acids by negative mode electrospray ionization. Rapid Commun. Mass Spectrom. 23(1), 171-180 (2009)

26. Mele, A., Mendichi, R., Selva, A., Molnar, P., Toth, G.: Noncovalent associations of cyclomaltooligosaccharides (cyclodextrins) with carotenoids in water. A study on the $\alpha$ - and $\beta$ - cyclodextrin $/ \mathrm{psi}$, psi-carotene (lycopene) systems by light scattering, ionspray ionization and tandem mass spectrometry. Carbohydr. Res. 337(12), 1129-1136 (2002)

27. Cescutti, P., Garozzo, D., Rizzo, R.: Effect of methylation of $\beta$ cyclodextrin on the formation of inclusion complexes with aromatic compounds. An ionspray mass spectrometry investigation. Carbohydr. Res. 302(1/2), 1-6 (1997)

28. Cescutti, P., Garozzo, D., Rizzo, R.: Study of the inclusion complexes of aromatic molecules with cyclodextrins using ionspray mass spectrometry. Carbohydr. Res. 290(2), 105-115 (1996)

29. Lamcharfi, E., Chuilon, S., Kerbal, A., Kunesch, G., Libot, F.: Electrospray ionization mass spectrometry in supramolecular chemistry: characterization of noncovalent cyclodextrin complexes. J. Mass Spectrom. 31(9), 982-986 (1996)

30. Ramanathan, R., Prokai, L.: Electrospray-ionization mass-spectrometric study of encapsulation of amino-acids by cyclodextrins. J. Am. Soc. Mass Spectrom. 6(9), 866-871 (1995)

31. Camilleri, P., Haskins, N.J., New, A.P., Saunders, M.R.: Analyzing the complexation of amino-acids and peptides with $\beta$-cyclodextrin using electrospray-ionization mass-spectrometry. Rapid Commun. Mass Spectrom. 7(10), 949-952 (1993)

32. Selva, A., Redenti, E., Zanol, M., Ventura, P., Casetta, B.: A study of $\beta$ cyclodextrin and its inclusion complexes with piroxicam and terfenadine by ionspray mass-spectrometry. Org. Mass Spectrom. 28(9), 983-986 (1993)

33. Zhang, M., Shi, Z., Bai, Y., Gao, Y., Hu, R., Zhao, F.: Using molecular recognition of $\beta$-cyclodextrin to determine molecular weights of lowmolecular-weight explosives by MALDI-TOF mass spectrometry. $J$. Am. Soc. Mass Spectrom. 17(2), 189-193 (2006)

34. Rekharsky, M.V., Inoue, Y.: Complexation thermodynamics of cyclodextrins. Chem. Rev. 98(5), 1875-1917 (1998)

35. Grünstein, D., Kikkeri, R., Maglinao, M., Collot, M., Barylyuk, K.V., Lapenies, B., Kamena, F., Zenobi, R., Seeberger, P.H.: Hexameric supramolecular scaffold senses bacteria by interactions with carbohydrates. Submitted to J. Am. Chem. Soc. (2011)

36. Zhao, Y., Schultz, N.E., Truhlar, D.G.: Design of density functionals by combining the method of constraint satisfaction with parametrization for thermochemistry, thermochemical kinetics, and noncovalent interactions. J. Chem. Theory Comput. 2(2), 364-382 (2006)

37. Grimme, S.: Semiempirical GGA-type density functional constructed with a long-range dispersion correction. J. Comput. Chem. 27(15), 1787-1799 (2006)

38. Dill, J.D., Pople, J.A.: Self-consistent molecular-orbital methods. 15. Extended Gaussian-type basis sets for lithium, beryllium, and boron. $J$. Chem. Phys. 62(7), 2921-2923 (1975)

39. Frisch, M.J., Trucks, G.W., Schlegel, H.B., Scuseria, G.E., Robb, M.A., Cheeseman, J.R., Scalmani, G., Barone, V., Mennucci, B., Petersson, G. A.,Nakatsuji, H., Caricato, M., Li, X., Hratchian, H.P., Izmaylov, A.F., Bloino, J.,Zheng, G., Sonnenberg, J.L., Hada, M., Ehara, M., Toyota, K., Fukuda, R.,Hasegawa, J., Ishida, M., Nakajima, T., Honda, Y.,
Kitao, O., Nakai, H., Vreven, T., Montgomery, J., J. A., Peralta, J.E., Ogliaro, F., Bearpark, M., Heyd, J.J., Brothers, E., Kudin, K.N., Staroverov, V.N., Kobayashi, R., Normand, J., Raghavachari, K., Rendell, A., Burant, J.C., Iyengar, S.S., Tomasi, J., Cossi, M., Rega, N., Millam, N.J., Klene, M., Knox, J.E., Cross, J.B., Bakken, V., Adamo, C., Jaramillo, J., Gomperts, R., Stratmann, R.E., Yazyev, O., Austin, A.J., Cammi, R., Pomelli, C., Ochterski, J.W., Martin, R.L., Morokuma, K., Zakrzewski, V.G., Voth, G.A., Salvador, P., Dannenberg, J.J., Dapprich, S., Daniels, A.D., Farkas, Ö., Foresman, J.B., Ortiz, J.V., Cioslowski, J., Fox, D.J.: Gaussian 09, revision a.1. 2009. Gaussian, Inc, Wallingford CT (2009)

40. Norman, P., Jiemchooroj, A., Sernelius, B.E.: Polarization propagator calculations of the polarizability tensor at imaginary frequencies and long-range interactions for the noble gases and $n$-alkanes. J. Chem. Phys. 118(20), 9167-9174 (2003)

41. Pettersen, E.F., Goddard, T.D., Huang, C.C., Couch, G.S., Greenblatt, D.M., Meng, E.C., Ferrin, T.E.: UCSF chimera - a visualization system for exploratory research and analysis. J. Comput. Chem. 25(13), 16051612 (2004)

42. Laskowski, R.A.: Surfnet-a program for visualizing molecular surfaces, cCavities, and intermolecular interactions. J. Mol. Graph. 13 (5), 323 (1995)

43. Kebarle, P., Verkerk, U.H.: Electrospray: from ions in solution to ions in the gas phase, what we know now. Mass Spectrom. Rev. 28(6), 898-917 (2009)

44. Hamilton, J.A., Sabesan, M.N.: Structure of a complex of cycloheptaamylose with 1-adamantanecarboxylic acid. Acta Crystallogr. B 38(12), 3063-3069 (1982)

45. Hamilton, J.A.: Structure of inclusion complexes of cyclomaltoheptaose (cycloheptaamylose): crystal structure of the 1-adamantanemethanol adduct. Carbohydr. Res. 142(1), 21-37 (1985)

46. Sánchez-Ruiz, X., Alvarez-Larena, A., Jaime, C., Piniella, J.F., Redondo, J., Virgili, A., Sanchez-Ferrando, F., Germain, G., Baert, F.: Molecular and crystal structure of the 1:1 complex of adamantanone with $\beta$-cyclodextrin. Supramol. Chem. 10(3), 219-223 (1999)

47. Zhao, Y., Truhlar, D.: The M06 suite of density functionals for main group thermochemistry, thermochemical kinetics, noncovalent interactions, excited states, and transition elements: two new functionals and systematic testing of four M06-class functionals and 12 other functionals. Theor. Chem. Acc. 120(1), 215-241 (2008)

48. Balabin, R.M., Lomakina, E.I.: Neural network approach to quantumchemistry data: accurate prediction of density functional theory energies. J. Chem. Phys. 131(7), 8 (2009)

49. Emsley, J.: Very strong hydrogen bonding. Chem. Soc. Rev. 9(1), 91124 (1980)

50. Barylyuk, K.V., Chingin, K., Balabin, R.M., Zenobi, R.: Fragmentation of benzylpyridinium "thermometer" ions and its effect on the accuracy of internal energy calibration. J. Am. Soc. Mass Spectrom. 21(1), 172177 (2010)

51. Gabelica, V., De Pauw, E.: Internal energy and fragmentation of ions produced in electrospray sources. Mass Spectrom. Rev. 24(4), 566-587 (2005)

52. Touboul, D., Jecklin, M.C., Zenobi, R.: Ion internal energy distributions validate the charge residue model for small molecule ion formation by spray methods. Rapid Commun. Mass Spectrom. 22(7), 1062-1068 (2008)

53. Chingin, K., Frankevich, V., Balabin, R.M., Barylyuk, K., Chen, H.W., Wang, R., Zenobi, R.: Direct access to isolated biomolecules under ambient conditions. Angew. Chem. Int. Ed. 49(13), 2358-2361 (2010)

54. Iribarne, J.V., Thomson, B.A.: Evaporation of small ions from charged droplets. J. Chem. Phys. 64(6), 2287-2294 (1976)

55. Thomson, B.A., Iribarne, J.V.: Field-induced ion evaporation from liquid surfaces at atmospheric pressure. J. Chem. Phys. 71(11), 44514463 (1979) 